\title{
Antitumor activity of ZD6474 in a metastatic orthotopic brain tumor model
}

\author{
DOO-SIK KONG ${ }^{1 *}$, MI-HYUN KIM ${ }^{1 *}$, JI-WON JEON ${ }^{1}$, SHI-YEON KIM ${ }^{1}$, MAENG SUP KIM ${ }^{2}$, \\ KYEUNG MIN JOO ${ }^{1}$, KWAN PARK ${ }^{1}$ and DO-HYUN NAM ${ }^{1}$ \\ ${ }^{1}$ Department of Neurosurgery, Samsung Medical Center and Samsung Biochemical Research Institute, \\ Sungkyunkwan University School of Medicine; ${ }^{2}$ Hanmi Pharmaceuticals Co., Seoul, Korea
}

Received January 7, 2008; Accepted February 28, 2008

\begin{abstract}
The objective of this study was to examine the antitumor effect of ZD6474, an orally available inhibitor of the vascular endothelial growth factor receptor-2 (VEGFR-2) and the epidermal growth factor receptor (EGFR), on tumor growth in an orthotopic metastatic brain tumor model. In order to determine the antitumor mechanism of ZD6474 treatment, in vitro and in vivo studies were performed. Human breast carcinoma cells (MDA-MB-435) were injected using direct intracranial (IC) inoculation $\left(5 \times 10^{5}\right.$ cells $\left./ 100 \mu \mathrm{l}\right)$ and internal carotid artery (ICA) injection $\left(5 \times 10^{4}\right.$ cells $\left./ 100 \mu 1\right)$ in Balb/c-nu female mice. Daily oral treatment with ZD6474 $(50 \mathrm{mg} / \mathrm{kg}$ ) was initiated on day 14 after the establishment of micrometastasis. Mice ( $\mathrm{n}=12$ per group) were sacrificed on day 28. Western blot analysis revealed that the autophosphorylation of EGFR and Akt was increasingly decreased with ZD6474 treatment in lung and brain endothelial cells and the MDA-MB-435 cell line. MTT assay also showed that the in vitro antitumor activity of ZD6474 was dependent on EGFR tyrosine kinase inhibition at a higher dose. Daily oral treatment with ZD6474 led to marked inhibition of metastatic tumor growth in the ICA injection and the direct IC inoculation models (median size $3.5 \mathrm{~mm}^{3}$, range $1.6-13.9 \mathrm{~mm}^{3}$ ) as compared to the control group (median size $62.4 \mathrm{~mm}^{3}$, range 11.5-206.9 $\mathrm{mm}^{3}$ ). These results suggest that simultaneous inhibition of both the EGFR and VEGFR-2 signaling pathways has a valuable therapeutic effect through its inhibition of the growth of metastatic brain tumors.
\end{abstract}

Correspondence to: Professor Kwan Park or Dr Do-Hyun Nam, Department of Neurosurgery, Samsung Medical Center, Sungkyunkwan University School of Medicine, 50 Ilwondong, Kangnamku, Seoul 135-710, Korea

E-mail: kwanpark@skku.edu; nsnam@skku.edu

${ }^{*}$ Contributed equally

Key words: vascular endothelial growth factor receptor, epidermal growth facor receptor, brain tumor

\section{Introduction}

In order to grow beyond a few millimeters in size, tumors must develop a functioning vascular network through which they can obtain essential nutrients and oxygen. In particular, they must develop a vascular supply if they are to grow to form clinically relevant metastases (1). Vascular endothelial growth factor (VEGF) and epidermal growth factor (EGF) have been strongly implicated in the metastatic spread and growth of tumors. VEGF is the most potent angiogenic factor identified to date (2).

ZD6474 is a novel agent that acts as a potent and reversible inhibitor of ATP binding to vascular endothelial growth factor receptor (VEGFR)-2 tyrosine kinases, and is considered the dominant signaling receptor governing endothelial cell (EC) permeability, proliferation and differentiation (3). In addition, it inhibits epidermal growth factor receptor (EGFR) tyrosine kinase activity. EGFR activation induces the activation of several downstream intracellular substrates, leading to mitogenic signaling and other tumor-promoting cellular activities. In human tumors, overexpression of this receptor correlates with a more aggressive clinical course (4). These data suggest that the blockade of both EGFR and VEGFR kinase activities and their respective downstream targets, such as Akt and phospho-Akt, could offer an attractive approach for the treatment of brain metastasis. ZD6474 is a novel orally available dual specific tyrosine kinase inhibitor of the ErbB (EGFR) and VEGFR receptors. It is a heteroaromatic-substituted anilinoquinazoline that acts as a potent and reversible inhibitor of ATP binding to VEGFR-2 tyrosine kinases. We hypothesized that inhibiting the phosphorylation of EGFR with ZD6474 should inhibit the tumor growth and metastasis of brain lesions. In the present study, we evaluated the effects of ZD6474 administered alone on the phosphorylation of these kinases, leading to cell growth arrest and apoptosis in vitro.

\section{Materials and methods}

Culture of the human breast carcinoma cell line. To study the biology of brain metastasis, human breast carcinoma MDA-MB-435 cells were maintained as a monolayer culture in Dulbecco's minimal essential medium (DMEM; Invitrogen, Burlington, ON, Canada) supplemented with $10 \%$ fetal bovine calf serum. 
A

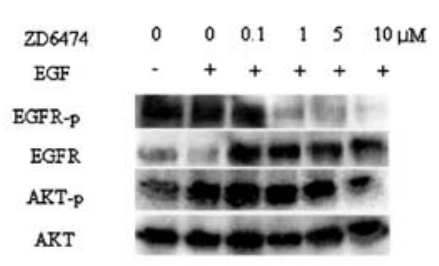

Lung EC

B

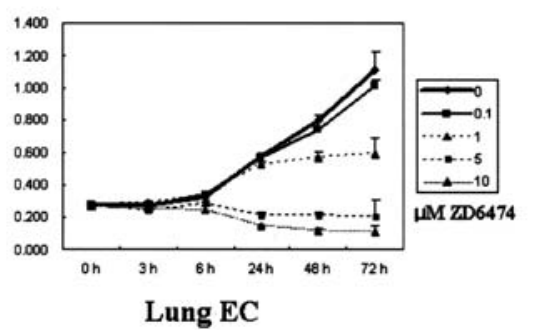

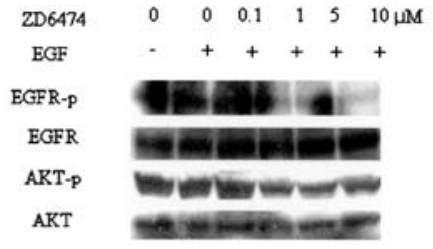

Brain EC (bEnd3)

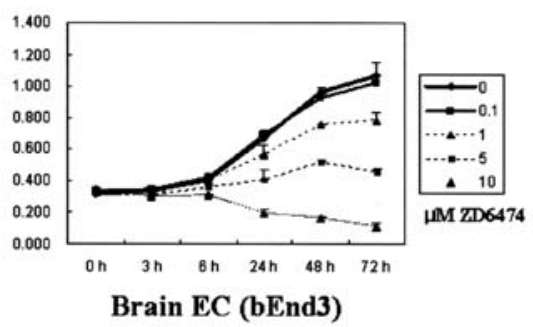

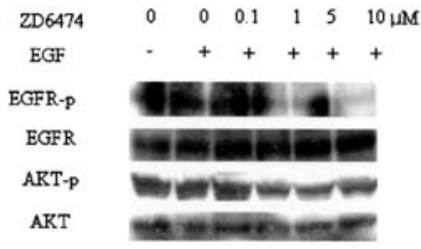

MDA-MB-435

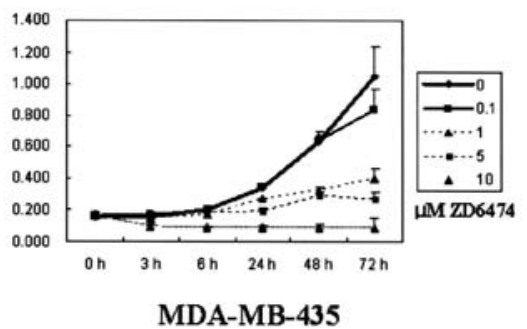

Figure 1. (A) Effect of ZD6474 treatment on EGF-induced phosphorylation of EGFR and Akt. Western blot analysis indicated that lung endothelial cells (ECs), brain ECs (bEnd3) and MDA-MB-435 breast carcinoma cells express constitutive levels of EGFR and Akt in cell cultures. In the absence of EGF in serum-free medium, EGFR and Akt remain phosphorylated at basal levels. The addition of EGF (1 or $10 \mathrm{ng} / \mathrm{ml}$ ) for 15 min to lung ECs, brain ECs and MDA-MB-435 breast carcinoma cells stimulates EGFR and Akt phosphorylation in a dose-dependent manner. The phosphorylation of EGFR and Akt was inhibited by pretreating cells with increasing concentrations of ZD6474. (B) The antiproliferative effects of ZD6474. MTT assay was performed to determine the effects of ZD6474 on the proliferation of cultured lung and brain ECs and MDA-MB-435 breast carcinoma cells. The dose-dependent growth inhibitory effects of various doses of ZD6474 are shown. The results are from a representative experiment performed in triplicate and repeated twice.

\section{In vitro study}

Measurement of cell proliferation. The antiproliferative activity of ZD6474 on lung ECs (provided by Dr Fidler, M.D. Anderson Cancer Center, Houston, TX), brain ECs (bEnd3; ATCC) and the MDA-MB-435 cell line growing in culture was determined using the tetrazolium-based colorimetric (MTT) assay. ZD6474 was provided by AstraZeneca Pharmaceuticals. Specifically, $5 \times 10^{4}$ cells (at passage $2-6$ ) were plated for $24 \mathrm{~h}$ into a 96 -well plate (1000 cells/well) and dosed with ZD6474. Cells were washed twice using $2 \%$ FBS medium and incubated for $0,3,6,24,48$ and $72 \mathrm{~h}$ with ZD6474. The cells were then incubated for $2 \mathrm{~h}$ in medium containing MTT, and then lysed in DMSO. The conversion of MTT to formazan by metabolically viable cells was monitored using a 96-well microtiter plate reader at an absorbance of 540 nm (Dynatech, Inc., Chantilly, VA).

Western blot analysis of phosphorylated EGFR/EGFR and phosphorylated Akt/Akt in lung ECs, brain ECs and the MDA-MB-435 cell line. The ability of ZD6474 to inhibit EGF-induced tyrosine phosphorylation of EGFR and Akt was determined in human lung and brain ECs and in the MDA-MB-435 cell line. Under serum-free conditions, the ECs showed a low level of autophosphorylation that was enhanced after exposure to recombinant human EGF for 15 min. Cells were plated onto a 6-well plate at a concentration of $5 \times 10^{4}$ cells per well and incubated in $10 \%$ FBS medium overnight. The next day, the cells were washed and incubated with serum-free medium for $24 \mathrm{~h}$. The study wells were treated with ZD6474 at a concentration of 0.1-10 $\mu \mathrm{M} / 1$, whereas the control wells were treated with DMSO for $1 \mathrm{~h}$. Then, cells were activated with recombinant human EGF (40 ng/ml) for 15 min, washed with phosphate-buffered saline (PBS) and scraped with lysis buffer as described previously. The proteins were resolved on $10 \%$ SDS-PAGE and transferred onto
$0.45 \mathrm{mmol} / 1$ polyvinylidene difluoride membranes. The membranes were probed overnight with the desired primary antibodies. After incubation with appropriate secondary antibodies, signals were visualized by the Super-Signal West Pico Chemiluminescent system from Pierce (Rockford, IL).

In vivo study. Female Balb/c-nu mice aged 6-8 weeks were cared for in accordance with the standards of the Samsung Biomedical Research Institute Council on Animal Care. Mice were anesthetized using a ketamine/xylazine mixture $(1.6 \mathrm{mg}$ ketamine and $0.08 \mathrm{mg}$ xylazine per $15 \mathrm{~g}$ body mass) administered by intraperitoneal injection (IP). To target cells to the brain, a suspension of $5 \times 10^{4}$ cells in $0.1 \mathrm{ml}$ of DMEM supplemented with $10 \%$ CS was injected into the internal carotid artery (ICA) of each mouse $(n=12)$. To determine the antitumor effect of the antiangiogenic agent, a suspension of $5 \times 10^{4}$ cells in $0.1 \mathrm{ml}$ of DMEM was injected into the brain by direct intracranial (IC) inoculation with the guidance of a stereotactic frame $(n=12)$. Breast carcinoma cells have been shown to form large highly vascularized metastases in the brain within two weeks of cell injection. To assess the biological effects of ZD6474 on pre-established brain metastases, tumor-bearing mice were treated with a single dose of ZD6474 $(50 \mathrm{mg} / \mathrm{kg}$, IP) on day 14, (after the establishment of micrometastases). ZD6474 solution was formulated in a vehicle of 5\% sodium carbonate and $80 \%$ PBS. Control mice were treated with vehicle alone, IP, on day 14 . Mice (n=12/group) were sacrificed on day 28 .

To assess the anti-metastatic effects of ZD6474 on developing brain metastases, mice were treated with ZD6474 $(0.1 \mathrm{ml}, 50 \mathrm{mg} / \mathrm{kg} /$ day) by oral gavage for 7 days (Fig. 1). ZD6474 solution was prepared in a vehicle of $1 \%$ polysorbate 80 in distilled water. Control mice were treated by gavage with this vehicle alone on the same schedule. Both control and 

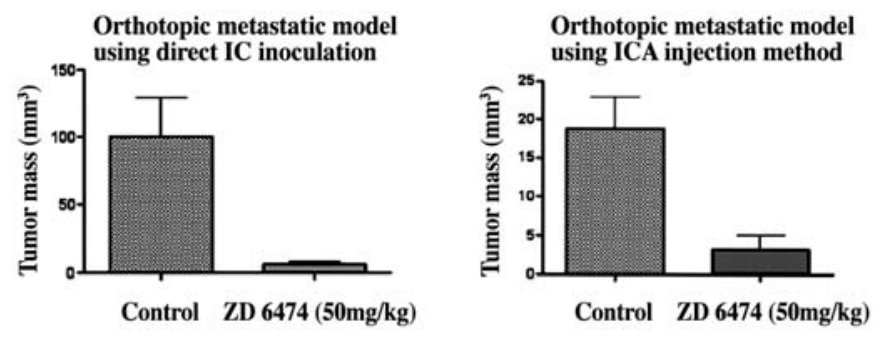

\begin{tabular}{lcc}
\hline & \multicolumn{2}{c}{ Tumor formation } \\
\cline { 2 - 3 } & Control & ZD6474 \\
\hline Intracranial inoculation model & $6 / 6$ & $6 / 6$ \\
\hline ICA injection model & $4 / 6$ & $3 / 6$
\end{tabular}

Figure 2. Tumor volumes in mice for each treatment group on observational day 28 in the MDA-MB-435 orthotopic model.

ZD6474-treated groups were sacrificed on day 28 to allow for comparison between groups.

Histology. To assess the growth and morphology of the developing metastases, formalin-fixed, paraffin-embedded brains were sectioned and stained with hematoxylin and eosin (H\&E). To assess the average size of the metastases, images from one section per mouse were captured using a color CCD camera and analyzed using Optimas ${ }^{\mathrm{TM}} 6.1$ image analysis software (Optimas Corp., Bothell, WA).

\section{Results}

ZD6474 inhibits the epidermal growth factor-induced phosphorylation of epidermal growth factor receptor and Akt in lung ECs, brain ECs and MDA-MB-435 cells. We investigated whether ZD6474 inhibits EGF-mediated growth and survival signaling pathways in lung ECs, brain ECs and MDA-MB-435 cells. These cells were starved of serum overnight, treated with increasing concentrations of ZD6474 for $1 \mathrm{~h}$, and then stimulated with EGF for $15 \mathrm{~min}$. Western blot analysis revealed that the autophosphorylation of EGFR and Akt was increasingly decreased with ZD6474 treatment in lung and brain ECs and in MDA-MB-435 cells growing in vitro in serum-free medium. The inhibition of EGFR phosphorylation required $1 \mu \mathrm{M} / 1$. The phosphorylated forms of Akt were down-modulated in cells treated with 5-10 $\mu \mathrm{M} / \mathrm{l}$. Total EGFR and Akt protein levels remained constant (Fig. 1A). MTT assay also revealed that the in vitro antitumor activity of ZD6474 was dependent on EGFR tyrosine kinase inhibition at a higher dose.

ZD6474 suppresses the in vitro proliferation of lung ECs, brain ECs and MDA-MB-435 cells. The growth of lung ECs, brain ECs and MDA-MB-435 cells in medium containing $2 \%$ FBS was inhibited by ZD6474 at a different dosage of $\mathrm{IC}_{50}$ for different incubation periods $\left(\mathrm{IC}_{50}: 1.25,2.56\right.$ and $0.89 \mu \mathrm{M}$ at $72 \mathrm{~h}$ ) (Fig. 1B).

The growth of metastatic brain tumor MDA-MB-435 cells in Balb/c-nu mice was inhibited by ZD6474. To assess the effect of ZD6474 on the in vivo growth of MDA-MB-435 cells in the brain, we adopted the established murine model of brain metastases by injecting MDA-MB-435 cells into the ICA or by direct IC inoculation using a stereotactic frame. ZD6474 markedly reduced the extent of metastatic brain tumors (Figs. 2 and 3). Treatment with ZD6474 led to marked inhibition of metastatic tumor growth in the IC inoculation model (median size $3.5 \mathrm{~mm}^{3}$, range $1.5-13.9 \mathrm{~mm}^{3}$ ) as compared with the control group (median size $62.4 \mathrm{~mm}^{3}$, range 11.5-206.9 $\mathrm{mm}^{3}$ ) (Fig. 2). We also found that treatment with ZD6474 was effective for reducing mass size in the direct IC inoculation model, the results of which were similar to those of the ICA injection model.
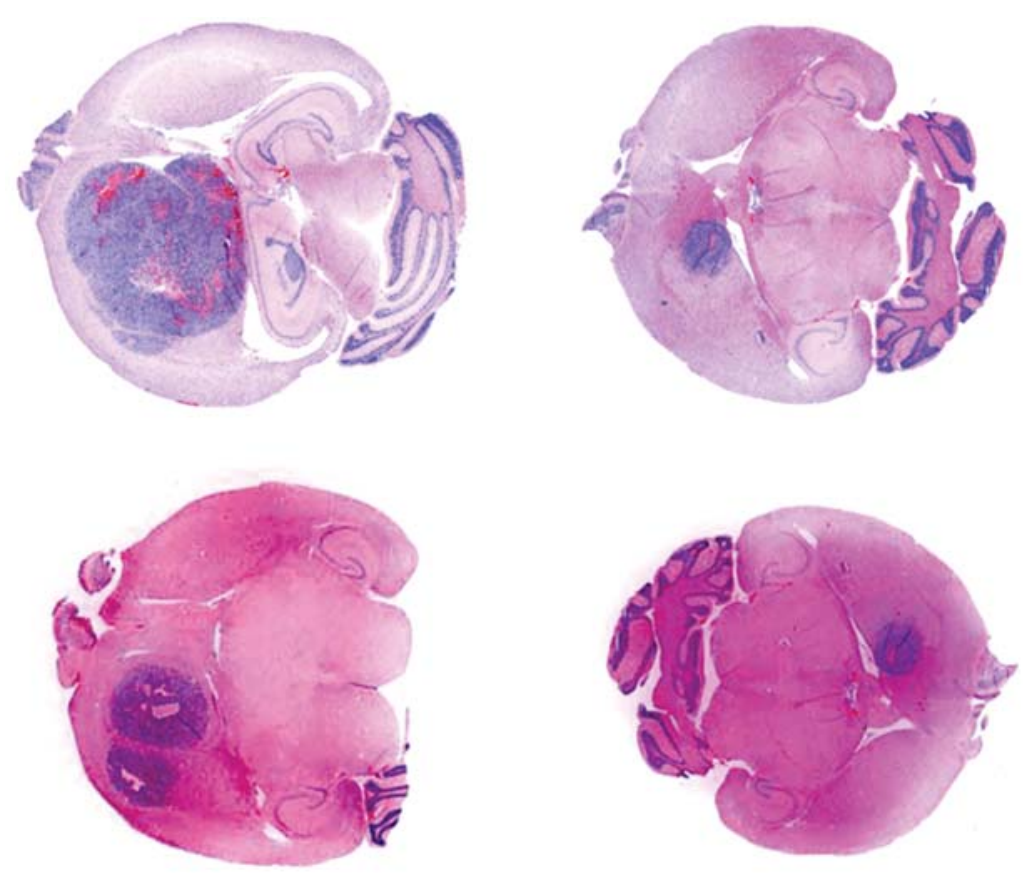

Figure 3. Pathology of the mouse brain. MDA-MB-435 breast carcinoma cells were implanted into the brain using direct IC inoculation and the ICA injection model. Mice were treated for 28 days with saline (control) or ZD6474 (50 mg/kg daily). 


\section{Discussion}

The growth of primary tumors and metastases is dependent on the development of new blood vessels. In addition, the aberrant disorganized nature of tumor vasculature provides a route whereby tumor cells can escape the primary tumor mass and disseminate to distant metastatic sites $(1,3,5,6)$. ZD6474 has been shown to inhibit the production of lung metastases in an orthotopic model of several carcinomas (7-15). These data demonstrate that ZD6474 has the potential to inhibit metastases through the prevention of primary tumor dissemination, as well as by inhibiting the growth of any secondary tumors that become established. Organ-tumor interactions at the site of the primary tumor and of metastasis are considered important determinants of tumor growth and development in humans. Taghian et al $(16,17)$ suggested that orthotopically implanted tumors may recapitulate the natural tumor setting more accurately than subcutaneous models. Theoretically, the ICA injection model may be superior to the direct IC inoculation model in view of physiologic and biological tumor settings for hematogenous brain metastases. However, in this study, we found that the ICA injection method showed a lower rate of tumor occurrence. It was therefore difficult to judge the therapeutic effect of the medication because of the rarity of tumor occurrence, the multiplicity of tumor formation and individual variation (Fig. 3). Instead, we found that the therapeutic effect of ZD6474 for reducing mass size was easily determined in the direct IC inoculation model, the results of which were compatible with those of the ICA injection model. Consequently, we suggest that the direct IC inoculation method has certain advantages over the ICA injection method, and can serve as a screening test for the evaluation of drug effect in orthotopic brain metastatic models.

In summary, these results suggest that simultaneous inhibition of both the EGFR and VEGFR2 signaling pathways has an effective therapeutic value in inhibiting the growth of metastatic brain tumors, and that the direct IC inoculation method can substitute for the ICA injection method in metastatic brain tumor models.

\section{Acknowledgements}

This study was supported by a grant from the Korea Research Foundation (2005-042-E00096).

\section{References}

1. Folkman J: Role of angiogenesis in tumor growth and metastasis. Semin Oncol 29: 15-18, 2002.

2. Neufeld G, Cohen T, Gengrinovitch S and Poltorak Z: Vascular endothelial growth factor (VEGF) and its receptors. FASEB J 13: 9-22, 1999.
3. Ferrara N: The role of vascular endothelial growth factor in pathological angiogenesis. Breast Cancer Res Treat 36: 127-137, 1995.

4. Mendelsohn J: Antibody-mediated EGF receptor blockade as an anticancer therapy: from the laboratory to the clinic. Cancer Immunol Immunother 52: 342-346, 2003.

5. Frederick B, Gustafson D, Bianco C, Ciardiello F, Dimery I and Raben D: ZD6474, an inhibitor of VEGFR and EGFR tyrosine kinase activity in combination with radiotherapy. Int $\mathrm{J}$ Radiat Oncol Biol Phys 64: 33-37, 2006.

6. Yokoi K, Kim SJ, Thaker P, Yazici S, Nam DH, He J, Sasaki T, Chiao PJ, Sclabas GM, Abbruzzese JL, Hamilton SR and Fidler IJ: Induction of apoptosis in tumor-associated endothelial cells and therapy of orthotopic human pancreatic carcinoma in nude mice. Neoplasia 7: 696-704, 2005.

7. Damiano V, Melisi D, Bianco C, Raben D, Caputo R, Fontanini G, Bianco R, Ryan A, Bianco AR, De Placido S, Ciardiello F and Tortora G: Cooperative antitumor effect of multitargeted kinase inhibitor ZD6474 and ionizing radiation in glioblastoma. Clin Cancer Res 11: 5639-5644, 2005.

8. Drevs J, Konerding MA, Wolloscheck T, Wedge SR, Ryan AJ, Ogilvie DJ and Esser N: The VEGF receptor tyrosine kinase inhibitor, ZD6474, inhibits angiogenesis and affects microvascular architecture within an orthotopically implanted renal cell carcinoma. Angiogenesis 7: 347-354, 2004.

9. Giannelli G, Azzariti A, Sgarra C, Porcelli L, Antonaci S and Paradiso A: ZD6474 inhibits proliferation and invasion of human hepatocellular carcinoma cells. Biochem Pharmacol 71: 479$485,2006$.

10. Hanrahan EO and Heymach JV: Vascular endothelial growth factor receptor tyrosine kinase inhibitors vandetanib (ZD6474) and AZD2171 in lung cancer. Clin Cancer Res 13: S4617-S4622, 2007.

11. Hoffmann S, Glaser S, Wunderlich A, Lingelbach S, Dietrich C, Burchert A, Muller $\mathrm{H}$, Rothmund $\mathrm{M}$ and Zielke A: Targeting the EGF/VEGF-R system by tyrosine-kinase inhibitors - a novel antiproliferative/antiangiogenic strategy in thyroid cancer. Langenbecks Arch Surg 391: 589-596, 2006.

12. Leenders WP, Küsters B, Verrijp K, Maass C, Wesseling P, Heerschap A, Ruiter D, Ryan A and De Waal R: Antiangiogenic therapy of cerebral melanoma metastases results in sustained tumor progression via vessel co-option. Clin Cancer Res 10: 6222-6230, 2004.

13. Yano S, Muguruma H, Matsumori Y, Goto H, Nakataki E, Edakuni N, Tomimoto H, Kakiuchi S, Yamamoto A, Uehara H, Ryan A and Sone S: Antitumor vascular strategy for controlling experimental metastatic spread of human small-cell lung cancer cells with ZD6474 in natural killer cell-depleted severe combined immunodeficient mice. Clin Cancer Res 11: 8789-8798, 2005.

14. Yokoi K, Sasaki T, Bucana CD, Fan D, Baker CH, Kitadai Y, Kuwai T, Abbruzzese JL and Fidler IJ: Simultaneous inhibition of EGFR, VEGFR, and platelet-derived growth factor receptor signaling combined with gemcitabine produces therapy of human pancreatic carcinoma and prolongs survival in an orthotopic nude mouse model. Cancer Res 65: 10371-10380, 2005.

15. Yokoi K, Thaker PH, Yazici S, Rebhun RR, Nam DH, He J, Kim SJ, Abbruzzese JL, Hamilton SR and Fidler IJ: Dual inhibition of epidermal growth factor receptor and vascular endothelial growth factor receptor phosphorylation by AEE788 reduces growth and metastasis of human colon carcinoma in an orthotopic nude mouse model. Cancer Res 65: 3716-3725, 2005.

16. Taghian A, De Vathaire F, Terrier P, Le M, Auquier A, Mouriesse H, Grimaud E, Sarrazin D and Tubiana M: Long-term risk of sarcoma following radiation treatment for breast cancer. Int J Radiat Oncol Biol Phys 21: 361-367, 1991.

17. Taghian A, Lespinasse F and Guichard ME: Radiosensitization by the combination of etanidazole (SR-2508) and pimonidazole (Ro 03-8799) in human tumor xenografts. Int J Radiat Oncol Biol Phys 21: 1535-1540, 1991. 